() И.А. Хрипун, С.В. Воробьев, Я.С. Аллахвердиева

Ростовский государственный медицинский университет, Ростов-на-Дону

ОБОСНОВАНИЕ. В современной литературе проводится поиск состояний, ассоциированных с дефицитом тестостерона (T), но данные о сочетании неалкогольной жировой болезни печени (НАЖБП) с гипогонадизмом и сахарным диабетом (СД) у мужчин крайне ограничены.

ЦЕЛЬ. Оценить влияние гипогонадизма на формирование и прогрессию НАЖБП у мужчин с СД 2 типа (СД2).

МЕТОДЫ. В исследование вошли 90 мужчин с СД2 (возраст 54 [49; 57] года). Пациентам проводили общеклинические исследования, изучение биохимических показателей (аланинаминотрансфераза (АЛТ), аспартатаминотрансфераза (AСТ), гамма-глутамилтранспептидаза (ГГТП), глюкоза, иммунореактивный инсулин, индекс НОМА, гликированный гемоглобин, липидограмма), выполняли иммуноферментные исследования (лютеинизирующий гормон, общий тестостерон (Т), глобулин, связывающий половые гормоны, резистин, адипонектин, лептин), производили магнитно-резонансную томографию с определением фракции жира печени.

РЕЗУЛЬтАТЫ. Пациенты были разделены на 2 группы: 1-я - 32 эугонадных пациента, 2-я - 58 мужчин с впервые установленным диагнозом гипогонадизма. Выявлено усиление инсулинорезистентности и компенсаторной гиперинсулинемии, а также гипертриглицеридемии у мужчин с гипогонадизмом по сравнению с эугонадными пациентами. Наряду с биохимическими признаками ухудшения функции печени, такими как увеличение концентраций печеночных ферментов (АЛТ на 24,5\% (p=0,02), ГГТП на 60,5\% (p=0,001)), коэффициента де Ритиса на 60,4\% (p=0,047), у мужчин с СД2 в сочетании с гипогонадизмом происходило повышение печеночной фракции жира печени, что в совокупности свидетельствует о прогрессии НАЖБП. Так, протонная плотность фракции жира в печени по данным МРТ в 1-й группе составила $4,12[2,25 ; 5,30] \%$, а во 2-й группе - 10,30 [7,78;14,44] \% (р<0,001). Это сопровождалось усилением продукции жировой тканью резистина в 2 раза и лептина в 12 раз $($ <<0,001) у пациентов 2-й группы по сравнению с пациентами 1-й группы.

ЗАКЛЮЧЕНИЕ. Сочетание СД2 с гипогонадизмом у мужчин приводит не только к нарушению углеводного и липидного обменов, но и к ухудшению функции печени - увеличению концентраций АЛТ, ГГТП, в сочетании с увеличением фракции жира печени. Предполагается, что повышение секреции жировой тканью лептина и резистина является связующим патогенетическим звеном в развитии нарушений углеводного и липидного обменов, НАЖБП и дефицита Т.

КЛЮЧЕВЫЕ СЛОВА: гипогонадизм; тестостерон; сахарный диабет; неалкогольная жировая болезнь печени; печень; андрогенный деричum

\title{
TESTOSTERONE DEFICIENCY AND NON-ALCOHOLIC FATTY LIVER DISEASE IN MEN WITH TYPE 2 DIABETES MELLITUS
}

(c) Irina A. Khripun, Sergey V. Vorobyev, Yanina S. Allakhverdieva

Rostov State Medical University, Rostov-on-Don, Russia

BACKGROUND: Current studies investigated diseases associated with testosterone (T) deficiency; however, data on the combination of non-alcoholic fatty liver disease (NAFLD) with hypogonadism and diabetes mellitus (DM) in men are extremely limited.

AIMS: To evaluate the effects of hypogonadism on the formation and progression of NAFLD in men with type 2 DM.

METHODS: The study included 90 men with type 2 DM [age 54 (49-57) years]. Patients underwent clinical examinations, biochemical analysis (alanine aminotransferase (ALT), aspartate aminotransferase, gamma-glutamyl transpeptidase (GGTP), fasting glucose, immunoreactive insulin, $\mathrm{HOMA}$ index, $\mathrm{HbA}_{1 c^{\prime}}$ lipid profile), immune enzyme analysis (luteinising hormone, total T, sex hormone binding globulin, resistin, adiponectin, leptin) and magnetic resonance imaging with liver fat fraction determination were performed. Patients were divided into two groups: 1-32 eugonadal patients and 2-58 men with newly diagnosed hypogonadism.

RESULTS: Increased insulin resistance, hyperinsulinemia, hypertriglyceridemia were observed in men with hypogonadism compared to eugonadal patients. Along with biochemical signs of impaired liver function, such as an increase in liver enzyme concentrations of ALT by $24.5 \%(p=0.02)$, GGTP by $60.5 \%(p=0.001)$, de Rytis coefficient by $60.4 \%(p=0.047)$, 
of men in the $2^{\text {nd }}$ group, the liver fat fraction also increased, which together indicates NAFLD progression. The proton density of the liver fat fraction according to MRI was $4.12[2.25-5.30] \%$ in the $1^{\text {st }}$ group and $10.30[7.78 ; 14.44] \%$ in the $2^{\text {nd }}$ group $(p=0.001)$. This was accompanied by an increase in fat production of resistin by 2 times and leptin by 12 times $(p<0.001)$ in patients of group 2 compared to 1 .

CONCLUSIONS: The combination of type $2 \mathrm{DM}$ with hypogonadism in men leads not only to deterioration of carbohydrate and lipid metabolism but also to disturbance of liver function: increased ALT, GGTP concentrations and liver fat. Increased secretion of leptin and resistin in the adipose tissue is assumed to be a pathogenetically associated with the development of carbohydrate and lipid metabolism disorders, NAFLD and T deficiency.

KEYWORDS: hypogonadism; testosterone; diabetes mellitus; nonalcoholic fatty liver disease; liver; androgen deficiency

Установлено, что проблема дефицита тестостерона (Т) имеет гораздо более широкий масштаб, чем предполагалось ранее, затрагивая немногим менее половины всего мужского населения в возрасте после 40 лет [1].

В последние годы все чаще обсуждается роль андрогенного дефицита у мужчин в патогенезе многих заболеваний, ассоциированных с метаболическим синдромом. Так, на сегодняшний день не вызывает сомнения тот факт, что гипогонадизм в несколько раз увеличивает риски развития ожирения, сахарного диабета 2 типа (СД2), артериальной гипертензии, дислипидемии [1]. Помимо этого, уже доказана взаимосвязь андрогенного дефицита с такими состояниями, как инсулинорезистентность, эндотелиальная дисфункция, дисбаланс цитокинов и эректильная дисфункция [2-4]. Причем все эти связи имеют двунаправленный характер, а заболевания взаимно отягощают друг друга, что позволяет рассматривать дефицит общего Т как дополнительный компонент метаболического синдрома $[5,6]$. Действительно, спектр нарушений различных органов и систем, возникающих на фоне недостаточности андрогенов у мужчин, необычайно широк, затрагивая не только половую систему, но и мозг, костно-мышечный аппарат, показатели углеводного и липидного обменов, сердечно-сосудистую систему. Долгое время противоречивыми оставались данные в отношении влияния Т на заболевания сердечно-сосудистой системы. Однако в последние годы преимущественное большинство исследований доказало значимость роли Т в формировании и развитии сосудистых рисков, а также положительного эффекта заместительной терапии Т у мужчин с гипогонадизмом $[7,8]$.

Вместе с тем исследователи выявляют новый фактор риска кардиоваскулярных заболеваний - неалкогольный стеатогепатоз $[9,10]$. Это, возможно, связано с тем, что неалкогольная жировая болезнь печени (НАЖБП) выявляется в 2-3 раза чаще у пациентов с ожирением и СД2 [11]. Столь тесная взаимосвязь неалкогольного стеатогепатоза с компонентами метаболического синдрома дает основание предположить, что дефицит Т может играть важную патогенетическую роль в развитии комплекса обсуждаемых заболеваний.

Однако в современной литературе имеются лишь единичные работы, посвященные влиянию дефицита Т на печень и показавшие, что его наличие способствует развитию и прогрессированию НАЖБП $[12,13,14]$. Это диктует необходимость поиска патогенетических механизмов, связывающих НАЖБП, гипогонадизм и ассоциированные с ними метаболические заболевания у мужчин.

\section{ЦЕЛЬ}

Оценить влияние гипогонадизма на формирование и прогрессию НАЖБП у мужчин с СД2.

\section{МЕТОДЫ}

\section{Дизайн исследования}

Проведено обсервационное одноцентровое одномоментное исследование.

\section{Критерии соответствия}

В исследование вошли мужчины в возрасте от 40 до 65 лет, имевшие СД2. Диагноз гипогонадизма устанавливался согласно диагностическим критериям Российской ассоциации эндокринологов (2017). Критериями исключения из исследования были первичные и вторичные формы гипогонадизма, что позволило сформировать когорту лиц со смешанным функциональным, или так называемым «неклассическим», гипогонадизмом.

Пациенты, вошедшие в исследование, характеризовались употреблением алкоголя не более 3 алкогольных единиц в неделю, что позволило исключить алкогольный генез поражения печени.

\section{Условия проведения}

Исследование проводилось на базе эндокринологического отделения клиники ФГБОУ ВО «Ростовский государственный медицинский университет» МЗ РФ.

Продолжительность исследования

Набор пациентов в исследование производили с 2018 по 2019 гг.

\section{Описание медицинского вмешательства}

Всем пациентам проводились общеклинические исследования, в том числе измерение антропометрических показателей и артериального давления. Больным проводили однократный забор венозной крови объемом 10 мл. Забор крови для исследований проводили строго натощак после 12-часового голодания. Для исследования биохимических показателей использовали свежую сыворотку крови, тогда как для проведения иммуноферментных анализов кровь центрифугировали и сыворотку замораживали при температуре $-20^{\circ} \mathrm{C}$. Изучали биохимические показатели крови пациентов, характеризующие функцию печени (аланинаминотрансферазу (АЛТ), аспартатаминотрансферазу (АСТ), с расчетом коэффициента де Ритиса, гамма-глутамилтранспептидазу (ГГТП)), а также состояние углеводного и липидного 
обменов (глюкозу и иммунореактивный инсулин (ИРИ) натощак с расчетом индекса инсулинорезистентности НОМА, гликированный гемоглобин А1с ( $\left.\mathrm{HbA}_{1 c}\right)$ и липидограмму. Помимо этого, определяли содержание половых гормонов - лютеинизирующего гормона (ЛГ), общего Т, глобулина, связывающего половые гормоны (ГСПГ) с последующим расчетом свободного Т, а также адипогормонов - резистина, адипонектина и лептина. После этого участникам исследования выполняли магнитно-резонансную томографию (МРТ) печени.

\section{Основной исход исследования}

В качестве основных конечных точек исследования были приняты показатели функции печени (АЛТ, АСТ, коэффициент де Ритиса, ГГТП), концентрации адипогормонов (резистина, адипонектина, лептина) и содержание фракции жира печени по данным МРТ.

\section{Анализ в подгруппах}

На основании проведенного клинического и лабораторного обследования, в зависимости от наличия или отсутствия гипогонадизма, пациенты были разделены на две группы.

- В 1-ю группу включили эугонадных пациентов с СД2.

- $\quad$ Bo 2-ю группу были включены мужчины с СД2 с впервые установленным диагнозом гипогонадизма.

\section{Методы регистрации исходов}

Биохимические исследования выполняли с использованием анализатора Bayer ADVIA 1650, определение $\mathrm{HbA}_{1 c}$ - анализатора Siemens Healthcare Diagnostics DCA 2000+. Иммуноферментные исследования проводили при помощи анализатора Zenyth 340. Для анализа содержания половых гормонов (общего Т, ГСПГ, ЛГ) использовали лабораторные наборы «Алкор-Био» (Россия). Показатель ИРИ определяли с помощью лабораторного набора Monobind Inc (США). На основании значений гликемии и инсулина определяли степень выраженности инсулинорезистентности с помощью расчета индекса НОМА по формуле:

НОМА = гликемия натощак (ммоль/л) $\times$ уровень инсулина (мкЕд/мл) / 22,5.

Коэффициент де Ритиса рассчитывали как отношение АСТ/АЛТ.

Изучение содержания адипогормонов проводили при помощи лабораторных наборов для иммуноферментного анализа: лептина - Bcm diagnostic Ilc (Германия), резистина - Biovendor laboratory (Чехия), адипонектина - eBioscience (Австрия).

Кроме того, всем пациентам на мультисрезовом спиральном рентгеновском компьютерном томографе Brilliance 64 Slice (Philips Medical Systems, Нидерланды) выполняли магнитно-резонансную томографию (МРТ) печени по методу Диксона с измерением доли печеночного жира, взвешенной по протонной плотности [15]. Данный метод считается единственным, производящим количественную оценку содержания жира печени [16].

Этическая экспертиза

Все пациенты подписали форму информированного согласия, одобренную Локальным этическим комитетом
Ростовского государственного медицинского университета (протокол №12/18 от 28.06.2018).

\section{Статистический анализ}

Статистическая обработка данных была проведена при помощи пакета прикладных программ Statistica 11.0. Bсе переменные были проверены на нормальность распределения с использованием критерия Колмогорова-Смирнова. Поскольку большинство показателей имели ненормальное распределение, данные представлены в виде медиан, нижнего и верхнего квартилей Me [Q25; Q75]. Значимость межгрупповых различий оценивалась с помощью U-критерия Манна-Уитни для двух независимых групп. Ковариантный анализ проводили при помощи модуля ANCOVA. Результаты считали статистически значимыми при значении для $\mathrm{p}<0,05$.

\section{РЕЗУЛЬТАТЫ}

Объекты (участники) исследования

В исследование вошли 90 мужчин с СД2, средний возраст которых составил 54 [49; 57] года, а длительность диабета - 6 [2; 10] лет. Первая группа была представлена 32 эугонадными пациентами с СД2. Вторая группа - 58 мужчинами с СД2 с впервые установленным диагнозом гипогонадизма. Группы были сопоставимы по возрасту, длительности течения и терапии СД2.

Сравнительная характеристика обследуемых групп представлена в табл. 1. Поскольку в основе деления пациентов с СД2 на группы лежали лабораторные критерии гипогонадизма в сочетании с клинической симптоматикой андрогенного дефицита, группы значимо ( $<<0,001)$ отличались по концентрациям как общего, так и свободного Т, а также ГСПГ, содержание которого было обратно пропорционально Т. При этом уровни ЛГ были средненормальными и сопоставимыми в обеих группах.

\section{Основные результаты исследования}

Клинические исследования выявили более выраженное абдоминальное ожирение у лиц с гипогонадизмом, что проявлялось в значениях индекса массы тела (ИМТ) на 6\% выше, а окружности талии (ОТ) на 6,9\% больше у пациентов 2-й группы по сравнению с обследуемыми 1-й группы $(p<0,01)$. Это сопровождалось большей окружностью бедер (ОБ) во 2-й группе по сравнению с 1-й ( $p<0,001)$, но при этом отношение ОТ/ОБ в группах было сопоставимо. Нужно обратить внимание на показатели артериального давления - было выявлено статистически значимое увеличение уровней как систолического давления (САД) ( $p=0,01)$, так и диастолического артериального давления (ДАД) $(p=0,02)$ во 2-й группе по сравнению с 1-й, тогда как частота сердечных сокращений была сопоставима.

Сравнительный анализ показателей углеводного обмена не выявил групповых отличий в уровнях гликемии натощак $(6,9[6,2 ; 7,8]$ ммоль/л - 1-я группа и 7,4 [6,8; 8,3] ммоль/л - 2-я группа) и $\mathrm{HbA}_{1 с}$ (7,6 [6,5; $7,9] \%$ и 7,7 [6,6; 7,9] \% соответственно). При этом значимыми были различия в концентрации ИРИ и индексе инсулинорезистентности НОМА. Нужно отметить, что пациенты обеих групп имели гиперинсулинемию, что 
Таблица 1. Сравнительная характеристика групп обследуемых

\begin{tabular}{lccc}
\hline \multicolumn{1}{c}{ Показатель } & $\begin{array}{c}\text { 1-я группа } \\
\text { (n=32) }\end{array}$ & $\begin{array}{c}\text { 2-я группа } \\
\text { (n=58) }\end{array}$ & p \\
\hline Возраст, годы & Клинические показатели & 0,18 \\
ИМТ, кг/м & $53,0[48 ; 56]$ & $54,0[49 ; 57]$ & 0,002 \\
ОТ, см & $29,9[29,2 ; 33,15]$ & $31,8[30,6 ; 34,0]$ & $<0,001$ \\
ОБ, см & $102,0[100,5 ; 107,5]$ & $109,0[102,5 ; 112,5]$ & $<0,001$ \\
ОТ/ОБ & $101,0[100 ; 103,5]$ & $106,0[100,5 ; 111]$ & 0,25 \\
САД, мм рт. ст. & $1,01[1,0 ; 1,02]$ & $1,02[1,0 ; 1,05]$ & 0,01 \\
ДАД, мм рт. ст. & $137[128 ; 150]$ & $148[132 ; 164]$ & 0,02 \\
ЧСС, уд/мин & $84[80 ; 90]$ & $90[80 ; 100]$ & 0,34 \\
\hline & $75[69 ; 82]$ & $75[69 ; 80]$ & $<0,001$ \\
\hline Общий тестостерон, нмоль/л & Уровни половых гормонов & $<0,001$ \\
ГСПГ, нмоль/л & $18,58[17,18 ; 22,05]$ & $8,46[6,50 ; 10,53]$ & $<0,001$ \\
Свободный тестостерон, & $24,4[26,1 ; 30,4]$ & 0,09 \\
пмоль/л & $24,2[21,9 ; 28,6]$ & $178[144 ; 203]$ & $5,2[4,0 ; 5,6]$ \\
ЛГ, мЕД/мл & $368[349 ; 413]$ & $5,6[4,3 ; 6,4]$ &
\end{tabular}

Примечания: ИМТ - индекс массы тела; ОТ - окружность талии; ОБ - окружность бедер; САД - систолическое артериальное давление; ДАД - диастолическое артериальное давление; ЧСС - частота сердечных сокращений; ГСПГ - глобулин, связывающий половые гормоны; ЛГ - лютеинизирующий гормон.

является характерной патогенетической особенностью СД2. Однако концентрация ИРИ натощак у гипогонадных пациентов (33,7 [23,3; 49,9] мМЕ/мл) была более чем в 1,5 раза выше ( $p=0,019)$, чем у эугонадных мужчин $(21,5[16,4 ;$ $34,6]$ мМЕ/мл). Индекс НОМА был вдвое больше $(p=0,009)$ у пациентов 2-й группы по сравнению с 1-й $(6,8$ [5,4; 9,6] против 11,4 [7,2; 16,2] во 2-й группе).

Концентрация общего холестерина в группах (6,3 [5,7; 6,9] ммоль/л и 6,3 [5,2; 7,2] ммоль/л), липопротеидов низкой плотности $(3,3[2,5 ; 3,8]$ ммоль/л и 3,3 $[2,7 ; 3,9]$ ммоль/л), а также липопротеидов высокой плотности $(1,4$ [1,2; 1,5] ммоль/л и 1,5 [1,3; 1,5] ммоль/л) у пациентов 1-й и 2-й групп соответственно значимо не отличалась. Однако содержание триглицеридов (ТГ) у пациентов с гипогонадизмом было на четверть выше, чем в группе эугонадных мужчин. Так, концентрация ТГ в 1-й группе составила 2,2 [1,9; 2,7] ммоль/л, а во 2-й группе - 2,7 [2,3; 3,8] ммоль/л ( $<<0,001)$.

Интерес представляют результаты анализа содержания адипогормонов, представленные на рис. 1, который выявил статистически значимое $(p<0,001)$ увеличение концентраций резистина в 2 раза и лептина в 12 раз у пациентов с дефицитом Т по сравнению с лицами, имевшими нормотестостеронемию. При этом различия в концентрациях адипонектина в группах были статистически не значимыми.

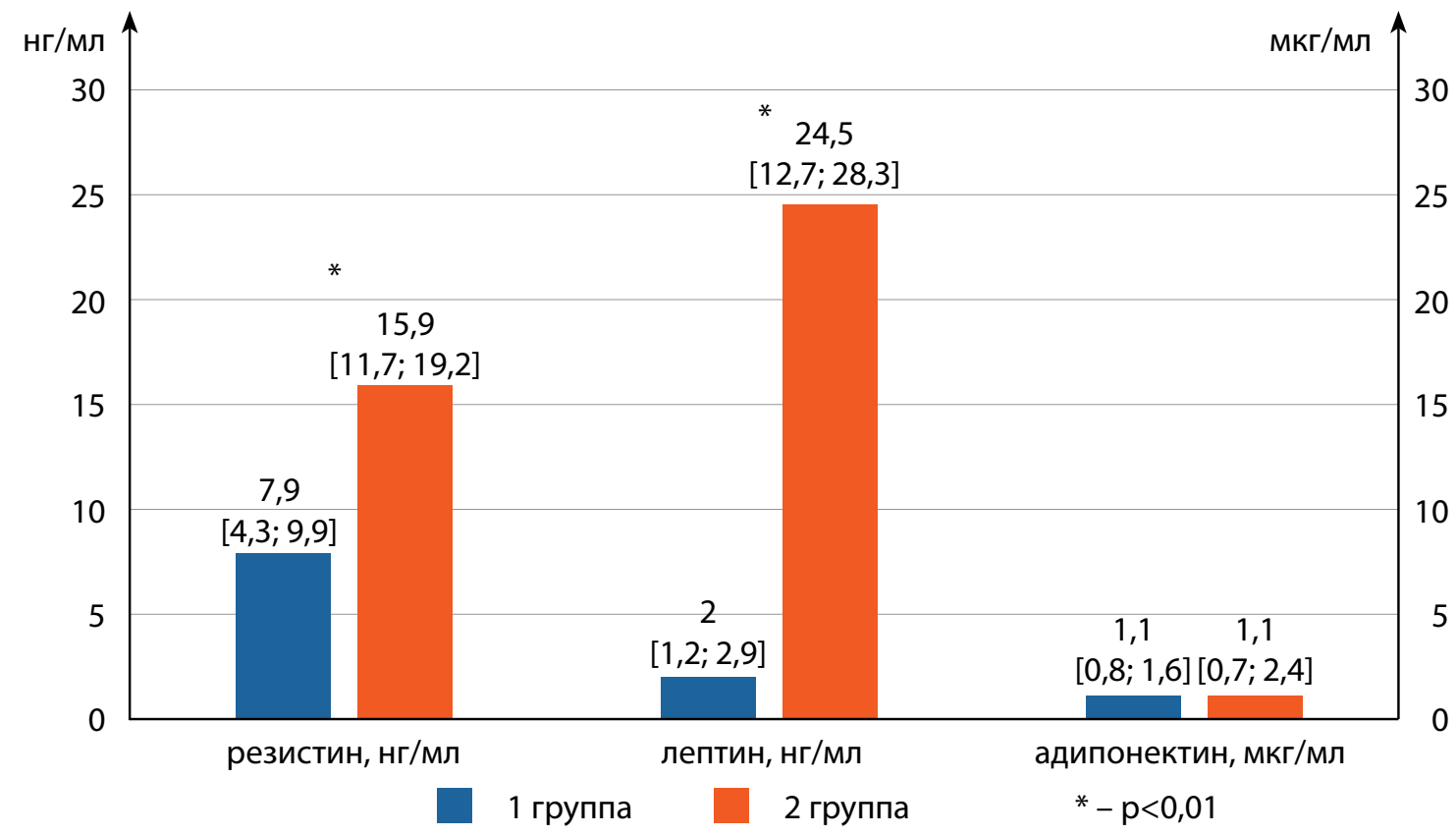

Рис. 1. Концентрации адипогормонов у мужчин с сахарным диабетом 2 типа в зависимости от наличия или отсутствия гипогонадизма. 
Активность ферментов печени у обследуемых представлена на рис. 2. При оценке функции печени не было выявлено достоверных различий в содержании общего и прямого билирубина, а также АСТ в группах. Однако у мужчин с гипогонадизмом активность АлТ была на 24,5\% выше $(p=0,02)$, а ГГТП - на 60,5\% выше $(p=0,001)$, чем у эугонадных пациентов. При этом известно, что именно ГГТП имеет максимальную чувствительность и специфичность в плане диагностики патологии печени. Это дополнялось статистически значимым различием в соотношении ферментов АСТ/АЛТ, характеризуемом коэффициентом де Ритиса, который был значимо выше $(p=0,047)$ на $60,4 \%$ у пациентов 2-й группы $(0,74[0,61 ; 0,89]$ единиц) по сравнению с 1 -й $(0,8[0,75 ; 0,94]$ единиц), что подтверждает поражение печени у обследуемых.

Выявленные изменения в лабораторных показателях дополняются данными инструментальных исследований, представленными на рис. 3. Протонная плотность фракции жира в печени по данным МРТ в 1-й группе составила 4,12 [2,25; 5,30]\%, а во 2-й группе - 10,30 [7,78; 14,44]\%. Таким образом, фракцияжирапечени была в 2,5 раза выше у мужчин с дефицитом Т по сравнению с эугонадными пациентами ( $<<0,001)$, что демонстрирует значимо большую выраженность неалкогольного стеатогепатоза у данной категории больных.

Отличие исследуемых групп по антропометрическим показателям требовало проведения ковариантного

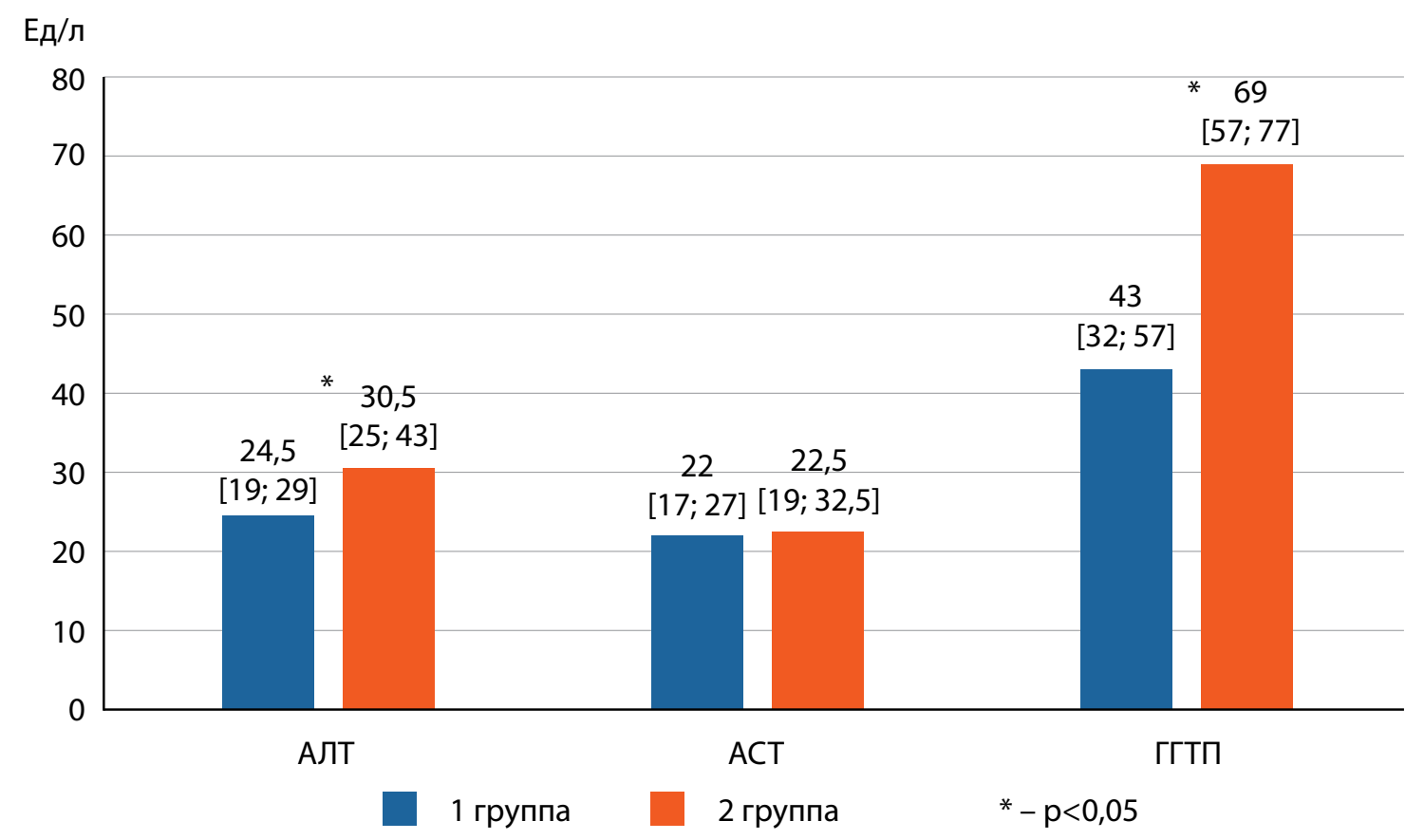

Рис. 2. Концентрации ферментов печени у мужчин с сахарным диабетом 2 типа в зависимости от наличия или отсутствия гипогонадизма.

$\%$

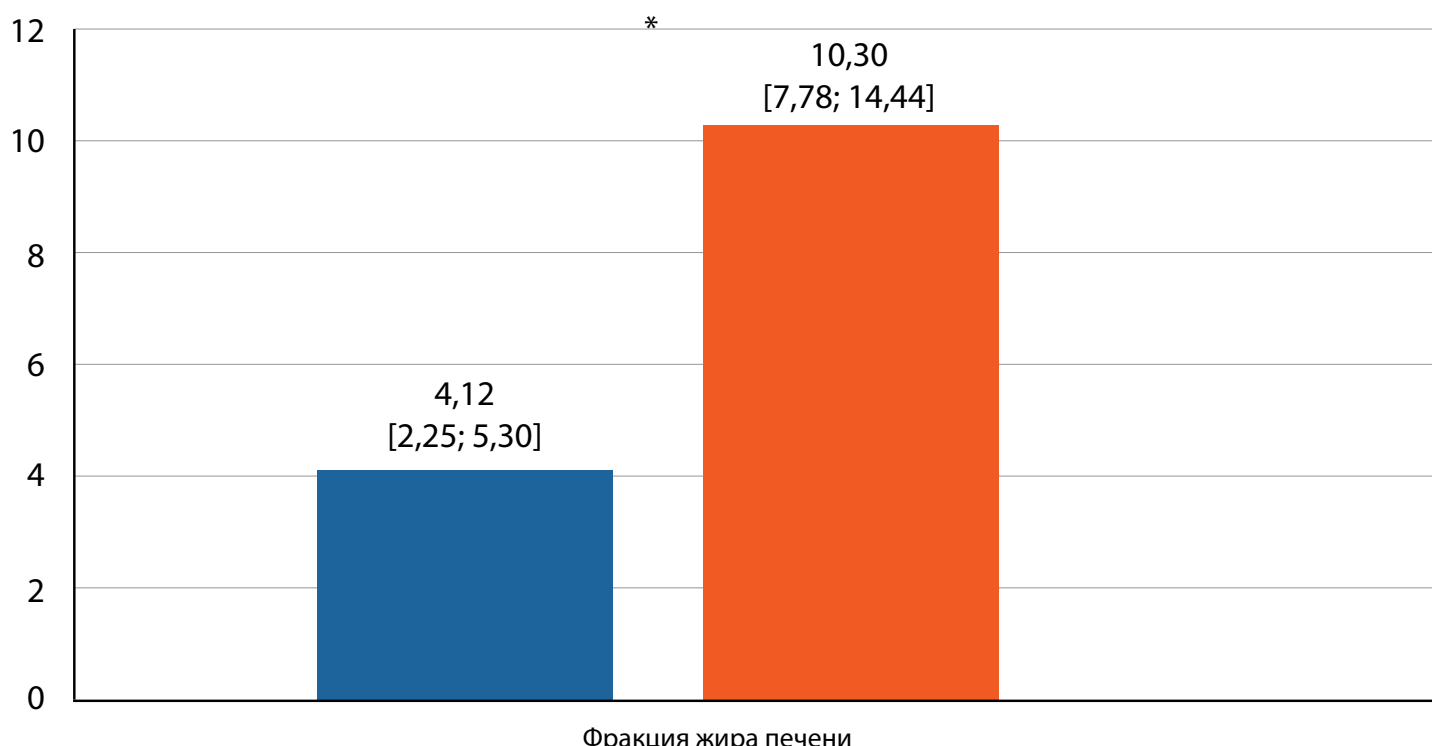

1 группа

2 группа

* $-p<0,001$

Рис. 3. Содержание фракции жира в печени у мужчин с сахарным диабетом 2 типа в зависимости от наличия или отсутствия гипогонадизма. 
анализа для оценки эффектов межгрупповых факторов и подтверждения значимости влияния на протонную плотность фракции жира печени именно дефицита Т, а не только выраженности ожирения. В связи с этим наличие гипогонадизма считали фактором, ИМТ и ОТ ковариантами, а количественное значение фракции жира печени - зависимой переменной. Ковариантный анализ установил, что влияние гипогонадизма на фракцию жира печени было статистически значимо с коррекцией как по ИМТ $(p=0,019)$, так и по ОТ $(p=0,002)$.

\section{Нежелательные явления}

В ходе проведенного исследования нежелательных явлений не возникало.

\section{ОБСУЖДЕНИЕ}

По данным многочисленных исследований $[1,3,5]$, гипогонадизм у мужчин сопровождается увеличением массы тела и преобладанием висцерального типа ожирения. Это было продемонстрировано и в нашей работе, где группа пациентов с гипогонадизмом имела более высокие показатели ИМТ, ОТ и ОБ по сравнению с эугонадными пациентами. Более того, были выявлены значимо более высокие показатели САД и ДАД во 2-й группе, что отражает более высокие кардиоваскулярные риски у мужчин с СД2 в сочетании с гипогонадизмом и дополняет общую картину метаболического синдрома у данной категории больных. Несмотря на отсутствие различий в гликемии натощак и концентрации $\mathrm{HbA}_{1 c}$ в группах, были выявлены значимо большие концентрации ИРИ и показатели индекса инсулинорезистентности НОМА у мужчин с дефицитом Т. Это позволяет считать выявленную гиперинсулинемию следствием инсулинорезистентности, ассоциированной с дефицитом Т у мужчин [17].

Именно инсулинорезистентность, приводя к гиперинсулинемии, ингибирует липолиз, параллельно активируя липогенез в печени, что и приводит к накоплению жира гепатоцитами под влиянием адипогормонов $[11,18]$. Кроме того, чем больше свободных жирных кислот накапливается клетками печени, тем выраженнее становится инсулинорезистентность самих гепатоцитов, стимулируя неконтролируемый глюконеогенез и продукцию глюкозы печенью. Таким образом, это объясняет взаимосвязь таких состояний, как СД2, НАЖБП и дефицит Т.

Одним из наиболее спорных вопросов по-прежнему остается влияние андрогенов на липидный обмен. Нами была выявлена значимо более высокая гипертриглицеридемия у пациентов с СД2 в сочетании с гипогонадизмом, что совпадает с результатами метаанализа [19]. На сегодняшний день пути воздействия андрогенов на липидный обмен продолжают изучаться. Установлено, что Т повышает активность печеночной липазы, осуществляя гидролиз ТГ и фосфолипидов, повышает активность гена скавенджер-рецептора SR-B1, способствующего избирательному захвату холестерина гепатоцитами, клетками Сертоли [20]. Следовательно, можно предположить, что дефицит Т приводит не только к дислипидемии, но и к бесконтрольному накоплению липидов клетками печени, способствуя тем самым развитию НАЖБП.
Не так давно изменилось отношение к гормонам, вырабатываемым жировой тканью, а именно резистину и лептину, которые из разряда регуляторов жирового и углеводного обменов были отнесены к категории предикторов сердечно-сосудистых заболеваний. Нами были выявлены достоверно более высокие концентрации резистина и лептина у пациентов с дефицитом Т по сравнению с лицами, имеющими нормотестостеронемию.

Известно, что, выделяясь адипоцитами, резистин не только угнетает инсулиноопосредованный захват глюкозы тканями-мишенями, по сути являясь антагонистом инсулина, но и способствует формированию пенистых клеток, активирует эндотелий сосудов, участвует в стимуляции процессов воспаления, в том числе и в печени, что позволяет его считать связующим звеном между ожирением, СД2, гепатостеатозом и атеросклерозом [21]. В то же время хорошо изученный с позиций его метаболических эффектов лептин также способствует отложению холестерина в макрофагах, стимулируя параллельно атерогенез и гепатостеатоз. Таким образом, мощная секреторная активность жировой ткани, выявленная в нашем исследовании у пациентов 2-й группы, непропорциональная мизерным межгрупповым отличиям в антропометрических параметрах, демонстрирует несомненную важность дефицита Т как одного из патогенетических факторов формирования метаболического синдрома у мужчин с СД2.

Важными являются данные, полученные при изучении функционального состояния и структуры печени. Так, установлено, что наличие гипогонадизма у мужчин с СД2 сопровождается повышением концентраций ферментов печени АЛТ, ГГТП и увеличением фракции жира печени по данным МРТ.

Подтверждением установленных взаимосвязей являются результаты ковариантного анализа, установившего статистическую значимость влияния гипогонадизма на фракцию жира печени с коррекцией по ИМТ и ОТ. Таким образом, можно утверждать, что у мужчин с СД2 и гипогонадизмом развитие НАЖБП достоверно связано именно с дефицитом T.

\section{Ограничения исследования}

Объем выборки исследования не был рассчитан до начала исследования, что ограничивает экстраполируемость результатов на популяцию в целом. Исследование проведено на группе пациентов, имеющих диагноз СД2, что ограничивает суждение о развитии НАЖБП у мужчин с гипогонадизмом без нарушений углеводного обмена.

\section{ЗАКЛЮЧЕНИЕ}

В современной литературе проводится поиск состояний, ассоциированных с дефицитом Т, но данные о сочетании НАЖБП с гипогонадизмом и СД у мужчин крайне ограничены. Результаты проведенного нами исследования выявили усиление инсулинорезистентности и компенсаторной гиперинсулинемии, а также гипертриглицеридемии у мужчин с гипогонадизмом по сравнению с эугонадными пациентами. Наряду с биохимическими признаками ухудшения функции печени, такими как уве- 
личение концентраций печеночных ферментов АЛТ, ГГТП, коэффициента де Ритиса, у мужчин с СД2 в сочетании с гипогонадизмом происходило повышение печеночной фракции жира печени, подтвержденное результатами инструментального исследования, что в совокупности свидетельствует о прогрессировании НАЖБП. Это сопровождалось усилением продукции жировой тканью адипогормонов резистина и лептина. Возможно, именно они являются связующим патогенетическим звеном в развитии таких состояний, как нарушения углеводного и липидного обменов, НАЖБП и дефицит Т.

\section{ДОПОЛНИТЕЛЬНАЯ ИНФОРМАЦИЯ}

Источник финансирования. Работа выполнена в рамках выполнения Государственного задания №14 «Функция печени у мужчин с сахарным диабетом 2 типа».

Конфликт интересов. Авторы заявляют об отсутствии финансовой заинтересованности и других потенциальных конфликтов интересов, связанных с публикацией настоящей статьи.

Участие авторов. Хрипун И.А., Воробьев С.В. - концепция и дизайн исследования, сбор материала, написание текста; Аллахвердиева Я.С. сбор материала, анализ результатов, написание текста. Все авторы внесли значимый вклад в проведение исследования и подготовку статьи, прочли и одобрили финальную версию статьи перед публикацией.

\section{СПИСОК ЛИТЕРАТУРЫ | REFERENCES}

1. Mulligan T, Frick MF, Zuraw QC, et al. Prevalence of hypogonadism in males aged at least 45 years: the HIM study. Int J Clin Pract. 2006;60(7):762-769. doi: https://doi.org/10.1111/j.1742-1241.2006.00992.x

2. Corona G, Bianchini S, Sforza A, et al. Hypogonadism as a possible link between metabolic diseases and erectile dysfunction in aging men. Hormones (Athens). 2015;14(4):569-578. doi: https://doi.org/10.14310/horm.2002.1635

3. Grossmann M, Wu FC. Male androgen deficiency: a multisystem syndrome. Asian J Androl. 2014;16(2):159-160. doi: https://doi.org/10.4103/1008-682X.122587

4. Хрипун И.А., Воробьев С.В., Пузырева В.П., и др. Дисфункция эндотелия как следствие андрогенного дефицита у мужчин с сахарным диабетом 2 типа // Современные проблемы науки и образования. - 2015. - №6. - С. 220. [Khripun IA, Vorob'ev SV, Puzyreva VP, et al. Dysfunction of endothelium, as a result of androgen deficiency in men with type 2 diabetes. Sovremennye problemy nauki i obrazovaniya. 2015;(6):220. (In Russ.)]

5. Ebrahimi F, Christ-Crain M. Metabolic syndrome and hypogonadism-two peas in a pod. Swiss Med Wkly. 2016;21(146):4283. doi: https://doi.org/10.4414/smw.2016.14283

6. Rastrelli G, Fillipi S, Sforza A. Metabolic syndrome in male hypogonadism. Front Horm Res. 2018;49:131-155. doi: https://doi.org/10.1159/000485999

7. Morgentaler A, Zitzmann M, Traish AM, et al. Fundamental concepts regarding testosterone deficiency and treatment: International Expert Consensus Resolutions. Mayo Clin Proc. 2016;91 (7):881-896. doi: https://doi.org/10.1016/j.mayocp.2016.04.007

8. Khripun I, Vorobyev S, Belousov I, et al. Influence of testosterone substitution on glycemic control and endothelial markers in men with newly diagnosed functional hypogonadism and type 2 diabetes mellitus: a randomized controlled trial. Aging male. 2019;22(4):241-249. doi: https://doi.org/10.1080/13685538.2018.1506918

9. Смирнова М.Д. Неалкогольная жировая болезнь печени неучтенный фактор риска атеросклероза // Русский медииинский журнал. Медицинское обозрение. - 2018. - Т. 2. — №4. - C. 8-11. [Smirnova MD. Non-alcoholic fatty liver disease as an unaccounted risk factor for atherosclerosis. RMJ. Medical Review. 2018;2(4):8-11. (In Russ.)]

10. Lee SB, Park GM, Lee JY, et al. Association between non-alcoholic fatty liver disease and subclinical coronary atherosclerosis: An observational cohort study. J Hepatol. 2018;68(5):1018-1024. doi: https://doi.org/10.1016/j.jhep.2017.12.012

11. Lucas C, Lucas G, Lucas N, et al. A systematic review of the present and future of non-alcoholic fatty liver disease. Clin Exp Hepatol. 2018;4(3):165-174. doi: https://doi.org/10.5114/ceh.2018.78120

12. Jaruvongvanich $V$, Sanguankeo A, Riangwiwat T, et al. Testosterone, sex hormone-binding globulin and nonalcoholic fatty liver disease: a systematic review and meta-analysis. Ann Hepatol. 2017;16(3):382-394 doi: https://doi.org/10.5604/16652681.1235481
13. Miyauchi S, Miyake T, Miyazaki M, et al. Free testosterone concentration is inversely associated with markers of liverfibrosis in men with type 2 diabetes mellitus. Endocr J. 2017;64(12):1137-1142 doi: https://doi.org/10.1507/endocrj.EJ17-0225

14. Аллахвердиева Я.С., Хрипун И.А., Воробьев С.В. Есть ли связь между андрогенным дефицитом и неалкогольной жировой болезнью печени у мужчин с сахарным диабетом 2 типа? В сб. тезисов VIII (XXV) Всероссийского диабетологического конгресса с международным участием «Сахарный диабет пандемия XXI века»; 28 февраля - 3 марта 2018 года. М.: ООО «УП ПРИНТ», 2018. - С. 260. [Allakhverdieva YaS, Khripun IA, Vorob'ev SV. Est' li svyaz' mezhdu androgennym defitsitom i nealkogol'noi zhirovoi bolezn'yu pecheni u muzhchin s sakharnym diabetom 2 tipa? Conference Proceedings of VIII (XXV) Russian diabetology congress with international

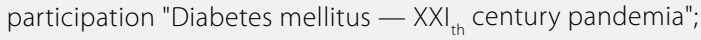
date 2018 Feb 28 - Mar 3. Moscow: OOO "UP PRINT"; 2018. P. 260 (In Russ.)]

15. Kim H, Taksali SE, Dufour S, et al. Comparative MR study of hepatic fat quantification using single-voxel proton spectroscopy, two-point dixon and three-point IDEAL. Magn Reson Med. 2008;59(3):521-527. doi: https://doi.org/10.1002/mrm.21561

16. Клинические рекомендации EASL-EASD-EASO по диагностике и лечению неалкогольной жировой болезни печени // Journal of Hepatology. Русское издание. - 2016. - Т.64. - С. 1388-1402. [Clinical recommendation EASL-EASD-EASO for the diagnosis and treatment of non-alcoholic fatty liver disease. Journal of Hepatology. Russian edition. 2016;64:1388-1402. (In Russ.)]

17. Коган М.И., Воробьев С.В., Хрипун И.А., и др. Тестостерон: от сексуальности к метаболическому контролю. Ростов на Дону: Феникс, 2017. — 239 с. [Kogan MI, Vorobyev SV, Khripun IA, et al. Testosterone: from sexuality to metabolic control. Rostov on Don: Phoenix; 2017. 239 p. (In Russ.)]

18. Мишина Е.Е., Майоров А.Ю., Богомолов П.О., и др. Неалкогольная жировая болезнь печени: причина или следствие инсулинорезистентности? // Сахарный duaбem. - 2017. - T. 20. - №5. - C. 335-342. [Mishina EE, Mayorov AY, Bogomolov PO, et al. Nonalcoholic fatty liver disease: cause or consequence of insulin resistance? Diabetes mellitus. 2017;20(5):335-342. (In Russ.)] doi: https://doi.org/10.14341/DM9372

19. Cai $X$, Tian $Y$, Wu T, et al. Metabolic effects of testosterone replacement therapy on hypogonadal men with type 2 diabetes mellitus: a systematic review and meta-analysis of randomized controlled trials. Asian J Androl. 2014;16(1):146-152. doi: https://doi.org/10.4103/1008-682X.122346

20. Vodo S, Bechi N, Petroni A, et al. Testosterone-induced effects on lipids and inflammation. Mediators Inflamm. 2013;2013:183041. doi: https://doi.org/10.1155/2013/183041

21. Park HK, Kwak MK, Kim HJ, et al. Linking resistin, inflammation, and cardiometabolic diseases. Korean J Intern Med. 2017;32(2):239-247. doi: https://doi.org/10.3904/kjim.2016.229 


\section{ИНФОРМАЦИЯ ОБ АВТОРАХ [AUTHORS INFO]}

Хрипун Ирина Алексеевна, к.м.н., доцент [Irina I. Khripun, MD, PhD, assistant professor]; адрес: Россия, 344022, г. Ростов-на-Дону, пер. Нахичеванский, д. 29 [address: 29, Nakhichevanski street, 344022 Rostov on Don, Russian Federation]; ORCID: https://orcid.org/0000-0003-0284-295X; eLibrary SPIN: 8630-4828; email: khripun.irina@gmail.com

Воробьев Сергей Владиславович, д.м.н., професcop [Sergey V. Vorobyev, MD, PhD, Professor]; ORCID: https://orcid.org/0000-0001-7884-2433; eLibrary SPIN: 9773-6100; e-mail: endocrinrostov@mail.ru Аллахвердиева Янина Сергеевна, аспирант [Yanina Y. Allakhverdieva, MD, PhD student]; ORCID: https://orcid.org/0000-0001-5514-9978; eLibrary SPIN: 8547-8020; e-mail: yana.allakhverdieva@yandex.ru

\section{ЦИТИРОВАТЬ:}

Хрипун И.А., Воробьев С.В., Аллахвердиева Я.С. Дефицит тестостерона и неалкогольная жировая болезнь печени у мужчин с сахарным диабетом 2 типа // Сахарный диабет. — 2019. — Т. 22. — №6. — С. $542-549$. doi: https://doi.org/10.14341/DM10232

\section{TO CITE THIS ARTICLE:}

Khripun IA, Vorobyev SV, Allahverdieva YS. Testosterone deficiency and non-alcoholic fatty liver disease in men with type 2 diabetes mellitus. Diabetes Mellitus. 2019;22(6):542-549. doi: https://doi.org/10.14341/DM10232 